\title{
Epstein-Barr virus-associated early gastric carcinoma with lymphoid stroma, accompanied with lymph node metastasis
}

\author{
TSUTOMU NAMIKAWA ${ }^{1}$, KAZUNE FUJISAWA ${ }^{1}$, ERI MUNEKAGE ${ }^{1}$, \\ MASAYA MUNEKAGE ${ }^{1}$, YUSUKE OKI ${ }^{2}$, HIROMICHI MAEDA ${ }^{3}$, HIROYUKI KITAGAWA ${ }^{1}$, \\ HIROSHI UETA ${ }^{4}$, MICHIYA KOBAYASHI ${ }^{3,5}$ and KAZUHIRO HANAZAKI ${ }^{1}$ \\ Departments of ${ }^{1}$ Surgery and ${ }^{2}$ Gastroenterology and Hepatology, Kochi Medical School, Kochi 783-8505; \\ ${ }^{3}$ Cancer Treatment Center, Kochi Medical School Hospital; ${ }^{4}$ Department of Gastroenterology \\ and Hepatology, Kochi Prefectural Hata Kenmin Hospital; ${ }^{5}$ Department of Human Health \\ and Medical Sciences, Kochi Medical School, Kochi 783-8505, Japan
}

Received May 5, 2017; Accepted November 28, 2017

DOI: $10.3892 / \mathrm{mco} .2018 .1567$

\begin{abstract}
The present case report presents a rare case of Epstein-Barr virus (EBV)-associated early gastric carcinoma with lymphoid stroma, accompanied by lymph node metastasis. A 61-year-old woman was referred to our hospital following observation of a gastric mass lesion that was initially diagnosed at a medical check-up. Esophagogastroduodenoscopy revealed a slightly elevated lesion with a central irregular depression in the middle third of the stomach. Endoscopic ultrasonography revealed a well-circumscribed hypoechoic mass located predominantly within the submucosa and the mucosa. Biopsy specimens of the lesion indicated the possibility of carcinoma with lymphoid stroma with a lymphoepithelial lesion. Abdominal contrast-enhanced computed tomography (CT) revealed a well-defined mass with homogeneous enhancement approximately $1.2 \mathrm{~cm}$ in diameter in the middle part of the stomach, demonstrating lymphadenopathy in the perigastric area at a maximum size of $1.4 \mathrm{~cm}$ in diameter. The patient underwent laparoscopic distal gastrectomy with regional lymphadenectomy due to suspicion of a gastric carcinoma with lymphoid stroma. Histopathological and immunohistochemical findings verified the diagnosis of carcinoma with lymphoid stroma, which were positive for EBV-encoded RNA (EBER-ISH) by in situ hybridization. There was one lymph node metastasis, which was in line with the CT imaging. Marked infiltration of lymphoid cells was observed in the tumor stroma, which were negative for EBER-ISH. The present case is informative of the pathological characteristics of this condition, and clinicians should recognize the features
\end{abstract}

Correspondence to: Dr Tsutomu Namikawa, Department of Surgery, Kochi Medical School, Kohasu, Oko-cho, Nankoku, Kochi 783-8505, Japan

E-mail: tsutomun@kochi-u.ac.jp

Key words: gastric cancer, Epstein-Barr virus, lymphoid stroma, lymphoepithelioma, gastrectomy, lymph node metastasis of this disease to make an accurate diagnosis and select the appropriate treatment.

\section{Introduction}

Epstein-Barr virus (EBV) is an established cause of various types of lymphoma including Burkitt lymphoma and Hodgkin's lymphoma, and nasopharyngeal carcinoma (1). Similar to Helicobacter pylori, EBV is accepted as an infective agent that plays an important pathogenic role in gastric cancer $(2,3)$. Gastric carcinoma with lymphoid stroma (GCLS) is a rare histological subgroup of gastric cancers, constituting approximately $1-4 \%$ of all gastric carcinomas. Approximately $80 \%$ of the reported GCLS cases are associated with an EBV infection $(1,4)$. To diagnose GCLS accurately, the immunohistochemical approach of the tumor was important in addition to sophisticated observation of the endoscopic findings, while standardized diagnostic criteria are lacking $(2,4)$. Early gastric cancer has favorable outcomes with curative resection including gastrectomy and endoscopic submucosal resection $(4,5)$. The current prognosis for GCLS is favorable due to its lower lymph node metastatic rate and a higher survival rate despite deep submucosal invasion of tumor cells.

In the present study, we report a case of EBV-associated early GCLS accompanying lymph node metastasis treated by laparoscopic distal gastrectomy.

\section{Case report}

A 61-year-old woman was referred to Kochi Medical School Hospital as a result of an evaluation of a gastric mass lesion that was initially diagnosed by a local medical doctor. Laboratory investigations, including serum carcinoembryonic antigen and cancer antigen 19-9 screening showed no significant abnormalities. Esophagogastroduodenoscopy (EGD) revealed an elevated lesion with a central irregularly depressed area in the posterior wall of the middle third of the stomach (Fig. 1A). The elevated lesion descended in a gradual slope to the surrounding mucosa when indigo carmine dye was used in chromoendoscopy (Fig. 1B). Endoscopic ultrasonography (EUS) revealed a 
well-circumscribed hypoechoic mass located predominantly within the submucosa and the mucosa (Fig. 2, arrow). Biopsy specimens of the lesion showed prominent lymphocyte infiltration with a lymphoepithelial lesion, suspected of being carcinoma with lymphoid stroma.

Abdominal contrast-enhanced computed tomography (CT) revealed a well-defined mass with homogeneous enhancement approximately $1.2 \mathrm{~cm}$ in diameter in the middle part of the stomach. The CT analysis also revealed lymphadenopathy in the perigastric area with a maximum size of $1.4 \mathrm{~cm}$ in diameter (Fig. 3, arrow). Double-contrast upper gastrointestinal imaging shows a round filling defect with central collection of Barium measuring $1.2 \mathrm{~cm}$ with a clear margin in the middle part of the stomach (Fig. 4, arrow).

As a result of these investigations we suspected that the patient had a gastric carcinoma with lymphoid stroma accompanying lymph node metastases. The patient underwent laparoscopic distal gastrectomy with reginal lymphadenectomy followed by Billroth I reconstruction. Macroscopic examination of the resected specimen showed a slightly elevated lesion-like submucosal tumor with a central depression measuring $1.2 \times 1.2 \mathrm{~cm}$ (Fig. 5, arrow).

Microscopic examination of the patient specimens showed a bulging mass consists of poorly differentiated adenocarcinoma. There was expansive growth into the submucosa that formed tubular structures and cancer nests with a submucosal depth of invasion of 4,000 $\mu \mathrm{m}$ (Fig. 6A and B). The tumor cells were positive for EBV-encoded RNA by in situ hybridization (EBER-ISH; Fig. 6C and D), and the expression of Ki-67 in cancer cells was $25 \%$. In addition, there was one lymph node metastasis in 13 dissected lymph nodes, which was detected as lymphadenopathy in the CT imaging but there was no lymphovascular infiltration. The lymph node station number with metastasis was 3 according to anatomical definition of Japanese classification of gastric carcinoma (6), which was $2.0 \mathrm{~cm}$ in diameter, consisting of diffuse spreading of adenocarcinoma in the lymph node (Fig. 7A and B). The results of the other immunohistochemical investigations of the primary gastric cancer and metastatic lymph node showed positive immunostaining for cytokeratin (AE1/AE3) and negative for CDX-2. Marked infiltration of lymphoid cells was observed in the tumor stroma and these were negative for EBER-ISH. The postoperative course was uneventful and the patient has been well without evidence of recurrence for two months following the operation.

\section{Discussion}

GCLS has distinct clinical characteristics that occur in old age and predominantly in males. These tumors arise in the cardia or middle portion of the stomach and have prominent lymphocyte infiltration, particularly in the submucosa $(7,8)$. EBER-ISH is the gold standard for determining EBV infection in histological sections. Carcinoma cells and dysplastic epithelial cells are positive for EBV, but not the normal epithelium or lymphoid stroma in EBV-associated GCLS. The estimate of EBV positivity in gastric cancer is $8.7 \%$ overall according to a meta-analysis, and the frequencies are not statistically different among different anatomical locations (1). Although EBV is considered to be the main cause

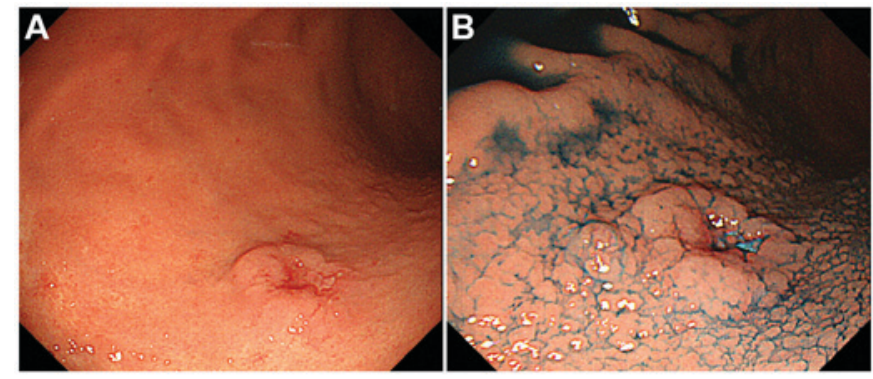

Figure 1. Esophagogastroduodenoscopy shows a slightly elevated lesion with central irregularly depressed area in the middle third of the stomach (A), which descends in a gradual slope to the surrounding mucosa using chromoendoscopy with indigo carmine dye (B).

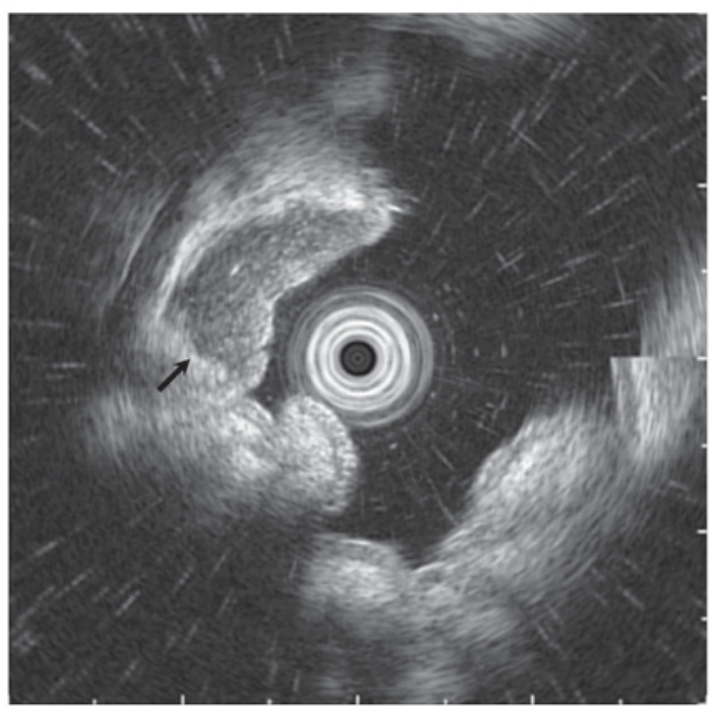

Figure 2. Endoscopic ultrasonography revealed a well-circumscribed hypoechoic mass located predominantly within the submucosa and the mucosa.

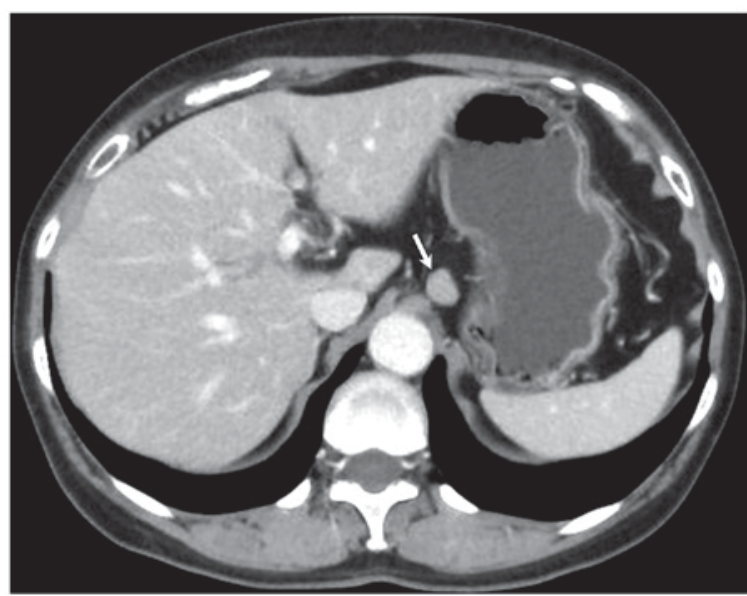

Figure 3. Abdominal computed tomography shows a well-defined ovoid mass with homogeneous enhancement measured in the perigastric area, with a maximum size of $1.4 \mathrm{~cm}$ in diameter (arrow).

of the lymphocytic response, the mechanism by which EBV contributes to the carcinogenesis of gastric mucosa has not been elucidated (9). 


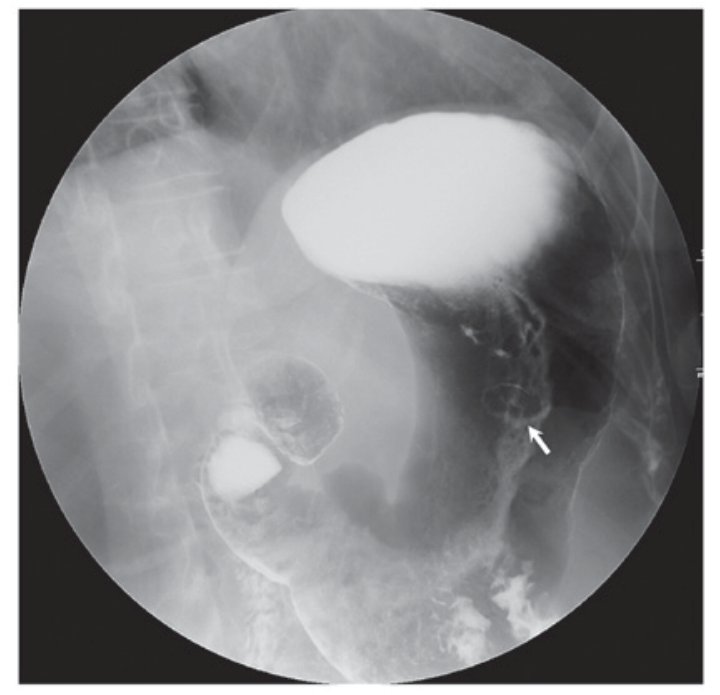

Figure 4. Double-contrast upper gastrointestinal imaging shows a round filling defect with central collection of Barium measuring $1.2 \mathrm{~cm}$ in diameter with a clear margin in the middle part of the stomach (arrow).

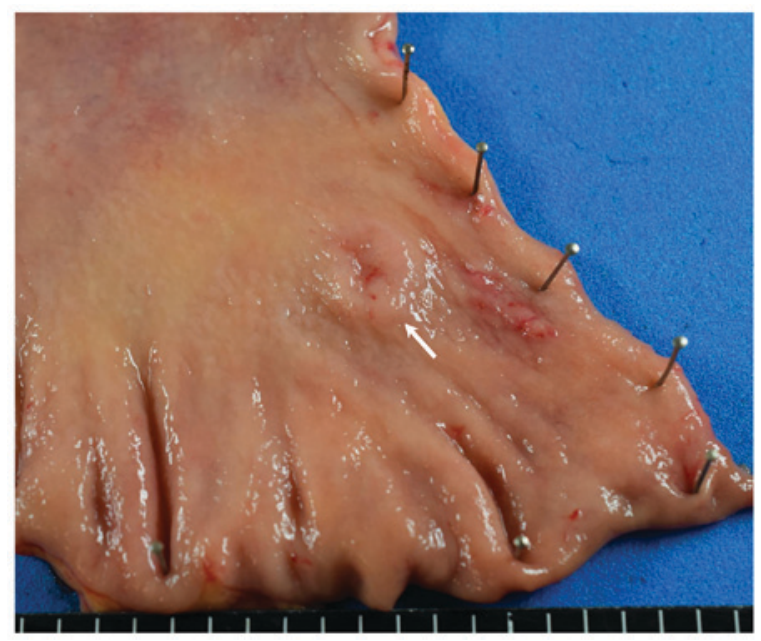

Figure 5. Gross appearance of the surgically resected specimen shows a slightly elevated lesion-like submucosal tumor with central depression measuring $1.2 \times 1.2 \mathrm{~cm}$ in the posterior wall side of middle third of the stomach (arrow).

The rate of metastasis to the lymph node in submucosal cancers with GCLS is significantly lower than in conventional early gastric cancer, especially those involving an EBV infection $(2,8)$. The spread of tumors through the gastric wall may be prevented by abundant lymphocytic reactions, which regulate the anti-tumor effect of immunity, resulting in a more favorable prognosis as a result of the immune response to the tumor $(8,9)$. An investigation of 41 GCLS in early gastric cancer reported that there was no lymph node metastasis if the submucosal depth of invasion was $\leq 2,000 \mu \mathrm{m}$ from the muscularis mucosa, while the incidence rate of lymph node metastasis was $28.6 \%$ if the SM depth of invasion was $>2,000 \mu \mathrm{m}$ (8).

Regarding the association between the sizes of gastric carcinoma and lymph node metastasis, Kim et al reported that there was no lymph node metastasis in the tumors with sizes $<1.0 \mathrm{~cm}$, and 2-dimensional tumor size was the only signifi-
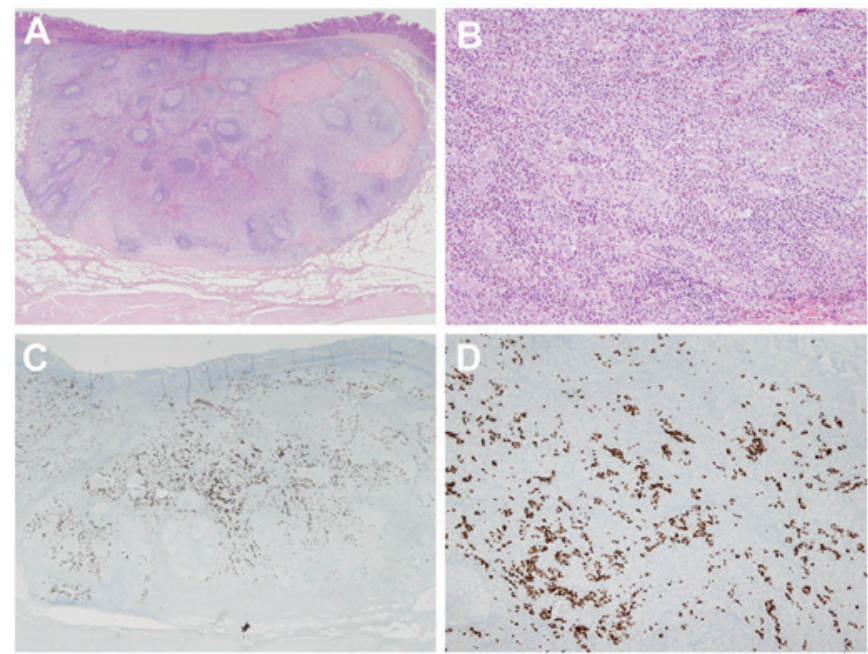

Figure 6. Histological examination of the resected specimen demonstrates a poorly differentiated adenocarcinoma with expansive growth into the submucosa [(A) low magnification; (B) high magnification]. Immunohistochemical examination shows that the tumor is positive for EBV-encoded RNA by in situ hybridization, whereas no signal can be seen in the lymphocytes. [(C) low magnification; (D) high magnification]. Stained with hematoxylin and eosin.
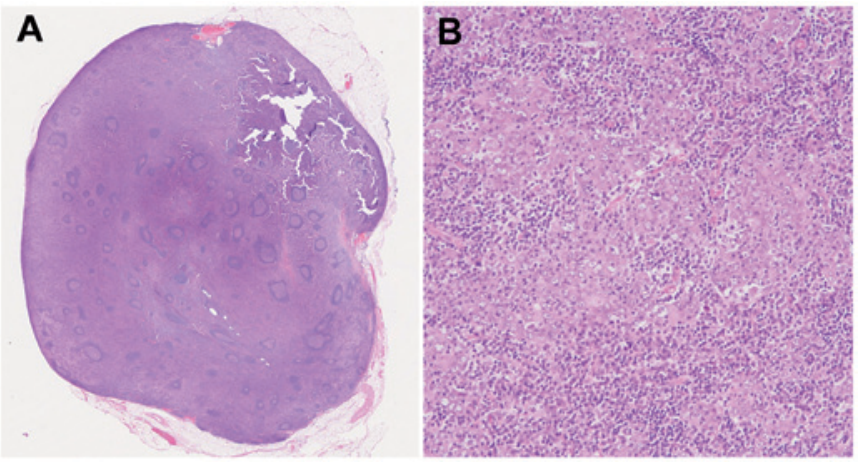

Figure 7. Histological examination of the lymph node which was $2.0 \mathrm{~cm}$ in diameter shows diffuse spreading of adenocarcinoma [(A) low magnification; (B) high magnification]. Stained with hematoxylin and eosin.

cant risk factor for lymph node metastasis in the analysis for 574 patients with differentiated minute submucosal cancer (10). Shin et al reported that tumor size was smaller in GCLS, $2.1 \mathrm{~cm}$, than in no-GCLS, $3.1 \mathrm{~cm}$, and only two patients with GCSL (3.4\%) showed lymph node metastasis in the analysis of 1696 patients with early gastric cancer (11). Therefore, the lymph node metastasis of small size GCLS as the present case seems to be extremely rare.

In the present case, it was difficult to detect the submucosal invasion during EGD without EUS, because the majority of the lesion was a bulging mass with abundant lymphoid stroma that was located in the submucosa by pathological investigation. Standard radical gastrectomy with regional lymphadenectomy is important, recommending in cases with submucosal invasion of tumor cells to a depth of more than $2,000 \mu \mathrm{m}$, regardless of tumor size. EUS seems to be an essential modality to estimate the depth of tumor invasion in the diagnostic and therapeutic management of GCLS. 
Since GCLS is a rare disease, clinicians should recognize the features of this entity to make an accurate diagnosis and select the appropriate treatment. Further studies and assessments of additional cases are required to establish standardized recommendation criteria and management for this entity.

\section{References}

1. Murphy G,PfeifferR,Camargo MC and Rabkin CS: Meta-analysis shows that prevalence of Epstein-Barr virus-positive gastric cancer differs based on sex and anatomic location. Gastroenterology 137: 824-833, 2009

2. Liang Q, Yao X, Tang S, Zhang J, Yau TO, Li X, Tang CM, Kang W, Lung RW, Li JW, et al: Integrative identification of Epstein-Barr virus-associated mutations and epigenetic alterations in gastric cancer. Gastroenterology 147: 1350-1362. e4, 2014.

3. Lim H, Park YS, Lee JH, Son DH, Ahn JY, Choi KS, Kim DH, Choi KD, Song HJ, Lee GH, et al: Features of gastric carcinoma with lymphoid stroma associated with Epstein-Barr virus. Clin Gastroenterol Hepatol 13: 1738-1744.e2, 2015.

4. Watanabe H, Enjoji $M$ and Imai T: Gastric carcinoma with lymphoid stroma. Its morphologic characteristics and prognostic correlations. Cancer 38: 232-243, 1976.

5. Namikawa T, Kitagawa H, Iwabu J, Okabayashi T, Sugimoto T, Kobayashi M, Hanazaki K. Clinicopathological properties of the superficial spreading type early gastric cancer. J Gastrointest Surg 14:52-57, 2010.

6. Japanese Gastric Cancer Association: Japanese classification of gastric carcinoma: III English edition. Gastric Cancer 14: 101-112, 2011.
7. Choi MG, Jeong JY, Kim KM, Bae JM, Noh JH, Sohn TS and Kim S: Clinical significance of gastritis cystica profunda and its association with Epstein-Barr virus in gastric cancer. Cancer 118: 5227-5233, 2012

8. Huh CW, Jung DH, Kim H, Kim H, Youn YH, Park H, Kim JW Choi SH, Noh SH and Kim JH: Clinicopathologic features of gastric carcinoma with lymphoid stroma in early gastric cancer. J Surg Oncol 114: 769-772, 2016.

9. Huang KH, Wang RF, Yang MH, Wu CW, Fang WL, Li AF, Chi CW and Kao HL: Advanced gastric cancer patients with lymphoid stroma have better survival than those without. J Surg Oncol 107: 523-528, 2013.

10. Kim TJ, Lee H, Min YW, Min BH, Lee JH, Kim KM, Kim MJ, Kim K, Rhee PL and Kim JJ: One-dimensional and 2-dimensional tumor size measurement for prediction of lymph node metastasis in differentiated early gastric cancer with minute submucosal invasion. Gastrointest Endosc 85: 730-736, 2017.

11. Shin DH, Kim GH, Lee BE, Lee JW, Ha DW, Jeon HK, Baek DH, Song GA, Ahn SJ and Park DY: Clinicopathologic features of early gastric carcinoma with lymphoid stroma and feasibility of endoscopic submucosal dissection. Surg Endosc: April 13, 2017. doi: 10.1007/s00464-017-5470-8. (Epub ahead of print). 